\title{
El efectode los videojuegos en variables sociales, psicológicas y fisiológicas en niños y adolescentes

\author{
The effect of playing videogames on social, psychological and physiological variables in children and \\ adolescents
}

\author{
José Moncada Jiménez y Yamileth Chacón Araya \\ Universidad de Costa Rica
}

\begin{abstract}
Resumen: El propósito de este trabajo será presentar evidencia científica acerca de los efectos del uso de los videojuegos en aspectos de la vida social de niños y adolescentes, así como posibles efectos psicológicos y fisiológicos generales. Se ha realizado una revisión de la literatura en bases de datos pertinentes y se han seleccionado investigaciones experimentales y meta analíticas de las cuales se extrajo información acerca de los efectos positivos y negativos de los videojuegos en niños y adolescentes. Se ha encontrado que la industria de los videojuegos es multimillonaria, y a pesar de que los videojuegos son populares en todo el mundo, la investigación científica sistemática es escasa y a veces, contradictoria. En algunos estudios se asocia el exceso de juego con efectos sociales y psicológicos negativos, como el aislamiento y la agresividad; mientras que en otros se asocia con el aprendizaje y reaprendizaje motor y la tenacidad para enfrentar situaciones de la vida real. En cuanto a efectos fisiológicos se ha encontrado que los juegos de video de naturaleza activa pueden ser promotores de un mayor gasto energético que los videojuegos de naturaleza pasiva; por lo que con una guía adecuada, podrían considerarse benéficos para combatir la epidemia global del sedentarismo y la obesidad. Se piensa que los juegos de video y todo lo relacionado con una pantalla serán parte de la vida de la población en el futuro, por lo que se deben realizar estudios sistemáticos para determinar los efectos de esta exposición a largo plazo.
\end{abstract}

Palabra clave: videojuegos, sedentarismo, agresividad, atención, gasto energético.

Abstract: The purpose of this manuscript will be to present scientific evidence regarding the effects of videogame playing on different aspects of the social life of children and adolescents, as well as the general potential psychological and physiological effects. A literature review from relevant databases has been performed, and experimental and meta-analytical studies have been scrutinized for positive and negative effects of videogames in children and adolescents. In general, it has been found that there is a billionaire videogame industry and yet, despite the worldwide popularity of videogames, research is still scarce and sometimes contradictory. Some research suggests a correlation between excess time video gaming on negative social and psychological aspects such as isolation and aggressive behavior; while other research suggests a positive association with motor learning, motor re-training and resilience. As far as physiological effects it has been reported that active videogames might promote higher energy expenditure than passive videogames; therefore, given an adequate parental instruction might provide videogames beneficial properties to combat the global epidemic of sedentary behavior and obesity. Videogames and everything related «to be» in front of a screen will be common to future generations, and therefore more systematic studies are required to determine the long-term exposure effects to these devices.

Key words: videogames, sedentary behavior, aggressive behavior, attention, energy expenditure.

\section{El juego en los niños}

Estes y Mechikoff (1999), han definido el verbo jugar, como una serie de actividades de ocio que se realizan de forma voluntaria, para diversión, y que es creativo y no tiene límites o reglas. Por su parte, los juegos son actividades que tienen una serie de reglas, en los que hay ganadores y perdedores y que tienden a ser específicos de acuerdo a la estructura del juego (e.g., coordinación ojo-mano) (Pellegrini, 2010). Las investigaciones apoyan el papel fundamental que el juego posee en el desarrollo socioemocional, cognitivo y físico del niño, así como en la regulación de las emociones, el fortalecimiento de las relaciones sociales con los miembros de su familia y amigos, en la atención, en la resolución de problemas, en la creatividad y en el desarrollo de destrezas motoras gruesas y finas, así como en su salud en general (Chacón Araya, 2005; Scarlett, Naudeau, Ponte, \& Salonius-Pasternak, 2004).

Para Bavelier, Green y Dye (2010), actualmente los niños y adolescentes se encuentran totalmente expuestos a la tecnología y a los medios de difusión electrónica tanto en la escuela como en sus hogares. Ya no es únicamente la televisión la que retiene la atención de los niños, sino también otros dispositivos como los DVDs de música, los videojuegos, internet, y los teléfonos celulares (móviles). En los Estados Unidos, se ha estimado que los niños se exponen a los medios electrónicos durante más de 7 horas por día (Strasburger, Jordan, \& Donnerstein, 2010), especialmente utilizando la T.V., escuchando música, usando los ordenadores y jugando videojuegos. Estos aparatos electrónicos se utilizan principalmente para la diversión y el ocio; sin embargo, también juegan un papel importante en el desarrollo del niño, especialmente en

Fecha recepción: 21-10-11 - Fecha envío revisores: 21-10-11 - Fecha de aceptación: 07-11-11 Correspondencia: José Moncada Jiménez

P.O.Box 239-1200 Pavas

San José, Costa Rica

E-mail: jose.moncada@ucr.ac.cr su aprendizaje y plasticidad cerebral, así como en su rendimiento académico, ya que la tecnología también ha sido introducida en el aula y para algunos podría ser una herramienta útil (Krotoski, 2010). Queda claro que estos medios pueden causar beneficios y perjuicios en el desarrollo cognitivo, social y conductual no solo de los niños (Ray \& Jat, 2010), sino también de los adultos (Rosser, Lynch, Cuddihy, Gentile, Klonsky, \& Merrell, 2007).

Quizás una de las mayores preocupaciones para los padres, es el rendimiento académico de sus hijos y su grado de actividad física (Moncada-Jiménez, 2005). Aunque la evidencia actual ha indicado que la actividad física proporcionada en las clases de educación física no empeora ni mejora el rendimiento académico, se conoce que el uso y abuso de la tecnología si puede perjudicar el rendimiento escolar (Mossle, Kleimann, Rehbein, \& Pfeiffer, 2010). Mossle et al. (2010), encuestaron a 5529 estudiantes y dieron seguimiento a otros 1157 estudiantes de escuela primaria y encontraron una asociación directa entre el tiempo que los estudiantes gastaban en juegos violentos y un pobre desempeño académico, especialmente en niños.

En el meta análisis de Cummings y Vandewater (2007), se ha encontrado que los niños y adolescentes que son asiduos usuarios de videojuegos leen en promedio un $30 \%$ menos que los niños que no los utilizan. También han indicado que el $34 \%$ dedica menos tiempo a hacer sus deberes escolares al compararlos con niños y adolescentes que no juegan videojuegos. Este hallazgo concuerda con el del Sharif, Wills y Sargent (2010), quienes encontraron varios mecanismos que explican el bajo rendimiento escolar. Estos investigadores entrevistaron telefónicamente a 6486 niños y adolescentes con edades entre 10 y 14 años, y han encontrado que la exposición prolongada a actividades frente a una pantalla (e.g., T.V., videojuegos) tenía un impacto negativo en el rendimiento académico. El mecanismo que podría explicar el hallazgo es la búsqueda de sensaciones placenteras, debido a que los adolescentes también miraban o jugaban videojuegos con alto contenido erótico. 
Mientras que en varias regiones del mundo, el uso de videojuegos se asocia a problemas sociales, físicos e incluso de tipo patológico (Choo, Gentile, Sim, Li, Khoo, \& Liau, 2010; Eagle, Gurm, Goldberg, DuRusselWeston, Kline-Rogers, Palma-Davis, Aaronson, Fitzgerald, Mitchell, Rogers, Bruenger, Jackson, \& Eagle, 2010), en un estudio canadiense no se encontró lo mismo. Carson, Pickett, y Janssen (2010), no encontraron una asociación entre el tiempo que los adolescentes pasaban jugando videojuegos con conductas de riesgo. Sin embargo, en la encuesta realizada a los 8215 adolescentes sí han encontrado que aquellos que usaban más los ordenadores tenían un $50 \%$ más de probabilidades de tener conductas de riesgo (e.g., uso de drogas, sexo sin protección, sedentarismo).

En Europa se realizó un estudio acerca de los hábitos de sedentarismo en adolescentes (Rey-Lopez, Vicente-Rodriguez, Ortega, Ruiz, Martinez-Gomez, De Henauw, Manios, Molnar, Polito, Verloigne, Castillo, Sjostrom, De Bourdeaudhuij, \& Moreno, 2010). Para ello, el equipo investigador obtuvo información de 3278 adolescentes entre 12 y 18 años de edad, en lo que se conoce como el estudio HELENA. Este proyecto incluía las ciudades de Atenas, Heracles, Dortmund, Gent, Lil, Pecs, Roma, Estocolmo, Viena, y Zaragoza. Entre los hallazgos más relevantes se ha obtenido que los adolescentes tuvieron comportamientos más sedentarios durante los fines de semana y que los hombres mayores de 15 años pasaban más tiempo viendo T.V. y jugando videojuegos que las mujeres. Esto indica que durante el tiempo de fin de semana, que teóricamente está más bajo el control de los padres, es cuando los adolescentes son más sedentarios. En otro estudio canadiense con una muestra menor $(\mathrm{n}=508)$, sí se ha encontrado que el uso excesivo (i.e., > $3 \mathrm{~h} /$ día) de videojuegos estaba asociado con tener padres sedentarios y con pocas reglas acerca del tiempo de uso de los aparatos electrónicos, por lo que un factor relevante para la utilización de la tecnología y la actividad física la definen los padres (He, Piche, Beynon, \& Harris, 2010).

Hoyos Cillero y Jago (2010), han resumido 71 investigaciones en idioma inglés publicadas entre los años 1980 y 2009. Los investigadores han encontrado una asociación entre los niños que pasaban más tiempo frente a las pantallas de T.V., ordenadores y videojuegos con variables como la edad, raza no predominantemente blanca, la cantidad de tiempo que la familia observaba T.V., y factores familiares como síntomas depresivos de la madre y una masa corporal elevada del padre. Por el contrario, los niños que no pasaban mucho tiempo frente a los dispositivos electrónicos se asociaban con un mayor ingreso económico de la familia y a reglas estrictas de los padres.

En los Estados Unidos incluso se han investigado las diferencias sexuales y raciales del uso de los videojuegos en el desarrollo de los niños. Hofferth (2010), concluye que las niñas se benefician más que los niños de edades entre 6 y 12 años, y que niñas y niños de raza negra se benefician más que los de raza blanca. También concluye que todas las niñas, independientemente de la raza, mostraron puntajes menores en la capacidad verbal, aunque las niñas de raza negra mejoraron su capacidad para resolver problemas. En los niños, el hallazgo más importante fue que el uso prolongado de los videojuegos se asociaba a una mayor cantidad de conductas agresivas y una menor capacidad para expresar sus sentimientos.

De acuerdo con Holtz y Appel (2011), en la adolescencia temprana se pasa más tiempo en internet y usando videojuegos que en cualquier otra etapa de la vida. En su estudio con 205 adolescentes austriacos con edades entre 10 y 14 años, los investigadores han encontrado que los videojuegos de apuestas en línea, que utilizaban internet y que se apropiaban del personaje (e.g., un asesino disparandobalas) eran variables predictoras de conductas como la agresión y la delincuencia. También han descrito que cuando el videojuego era más acerca de interpretar un personaje (《role-playing») se convertía en un factor predictor de aislamiento y ansiedad en el adolescente. Por ello, el juego también puede ser patológico, especialmente porque actualmente existen formas para inducir a las personas a gastar su dinero en línea (i.e., por internet), como por ejemplo, las apuestas, en donde los juegos son accesibles y atractivos para los jóvenes (King, Delfabbro, \& Griffiths, 2010).

\section{El videojuego}

De acuerdo con «The Entertainment Software Association» de los Estados Unidos (2011), la industria de los videojuegos produjo en el año 2009 aproximadamente $\$ 10.5$ billones, que en el $67 \%$ de los hogares juegan videojuegos, el jugador promedio tiene 24 años y que ha jugado durante al menos 12 años. También han indicado que la edad promedio del comprador de juegos es de 40 años, el $40 \%$ de los jugadores son mujeres y que personas mayores de 50 años representaron el $26 \%$ de los jugadores en el año 2010.

Un videojuego es un programa informático en el que el usuario o jugador mantiene una interacción a través de imágenes que aparecen en un dispositivo que posee una pantalla que puede variar de tamaño. Como todo juego, posee reglas y un sistema de recompensa, de manera que existe un estímulo implícito para intentar ganar. En un videojuego se puede competir contra la propia máquina (i.e., software o programa de la máquina) o contra otros adversarios. Un videojuego puede ser controlado únicamente por los dedos, en una interacción óculo manual, o con ayuda de otras partes del cuerpo (e.g., juegos de bailes) u otros instrumentos adicionales (e.g., guitarra, rifle, pistola). Actualmente, se desarrollan videojuegos que pueden ser activados por la voz o el movimiento, por lo que no es necesario el uso de otros accesorios.

Para Belli y López Raventós (2008), los videojuegos vienen en varias presentaciones, como por ejemplo, las consolas personales dinámicas, como por ejemplo el «Game boy» de Nintendo ${ }^{\circledR}$ y el «Play Station Portable» (PSP) de Sony ${ }^{\circledR}$, los cuales son para un solo jugador y pueden ser trasladados a cualquier lugar. También existen las consolas estáticas tradicionales («Arcade games»), que son de mucho mayor tamaño, que se encuentran generalmente en un parque de juegos y se tiene que introducir una moneda para poder utilizarla. Sin embargo, ahora existe una versión miniaturizada, pero aún estática, que son las consolas de menor tamaño, pero que brindan portabilidad. Por ejemplo, las consolas Wii y GameCube de Nintendo ${ }^{\circledR}$, el Xbox 360 de Microsoft $^{\circledR}$, y el Play Station 3 de Sony ${ }^{\circledR}$. También existen los videojuegos para ordenador, que básicamente se adquieren por medio de un disco compacto o actualmente se pueden comprar en línea. También hay dispositivos adicionales que se pueden conectar con cables o por medio de una señal infrarroja con los dispositivos (e.g., guitarras, plataforma 〈fit», alfombra para bailar, y otros). Finalmente, hay juegos que se pueden descargar hasta dispositivos como teléfonos celulares.

Existe un sistema de clasificación de los juegos de acuerdo con su contenido y que de alguna forma nos permite conocer el público al que supuestamente están dirigidos, así como los posibles riesgos de su utilización (Entertainment Software Rating Board, 2010). Esta clasificación se realiza por medio de letras de la siguiente manera:

1. EC («early childhood»): son videojuegos con contenidos temáticos para niños d» 3 años. No contiene material que los padres puedan considerar inapropiado.

2. E («everyone»): son videojuegos para niños e» 6 años. Estos juegos presentan dibujos animados y fantasías, violencia muy moderada y uso poco frecuente de lenguaje moderado.

3. E10+ («everyone $10+»)$ : son videojuegos con contenidos temáticos para niños e» 10 años. Estos juegos presentan más dibujos animados, fantasías y violencia moderada, uso frecuente de lenguaje moderado y temas mínimamente sugestivos.

4. T («teen»): son videojuegos con contenidos temáticos para niños, preadolescentes y adolescentes e» 13 años. Los videojuegos en esta categoría pueden contener violencia, temas sugestivos, humor crudo, alguna sangre, simulación de apuestas, y uso poco frecuente de lenguaje fuerte.

5. M («mature»): son videojuegos con contenidos temáticos adolescentes e» 17 años. Los videojuegos en esta categoría pueden presentar violencia intensa, imágenes sangrientas, contenido sexual, y lenguaje fuerte.

6. AO («adults only»): son videojuegos con contenidos temáticos sólo para personas e» 18 años. Los videojuegos en esta categoría pueden presentar escenas prolongadas de violencia intensa y contenido sexual gráfico explícito y desnudez. 
7. RP («rating pending»): significa que el videojuego no ha sido clasificado aún.

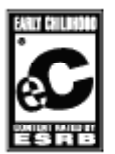

AGE $3+$

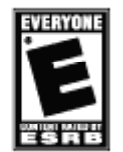

AGE $6+$
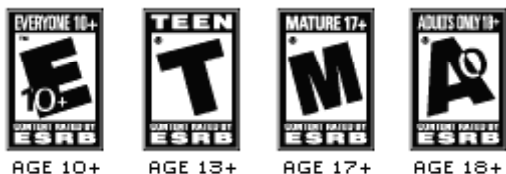

Fuente: Entertainment Software Rating Board (2010).

En el año 2009, el 48\% de todos los juegos vendidos en USA tuvieron una clasificación $\langle\mathbf{E} », 22 \%\langle\mathbf{T} », 17 \%\langle\mathbf{M} »$, y $12 \%\langle\mathbf{E} 10+»$ (The Entertainment Software Association, 2011).

\section{Videojuegos pasivos y activos}

Los videojuegos pasivos involucran básicamente el uso de la coordinación óculomanual para interactuar con el dispositivo electrónico. Estos juegos generalmente se utilizan en una posición pasiva (e.g., la persona sentada en un sillón) y con pocos movimientos del cuerpo. Son los juegos que más críticas reciben porque promueven el sedentarismo y el consumo de alimentos de poco valor nutritivo. Por su parte, los videojuegos activos son juegos electrónicos que les permiten a los jugadores interactuar físicamente utilizando sus manos, brazos, piernas o el cuerpo completo, con imágenes que aparecen en una pantalla o dispositivo de salida. El éxito en el juego depende, entre otros factores, del movimiento que se realice frente a una cámara, un sensor infrarrojo, un láser, una alfombra sensible a la presión o un ergómetro modificado (Foley \& Maddison, 2010).

Levac, Pierrynowski, Canestraro, Gurr, Leonard y Neeley (2010), han realizado un elegante estudio con 38 niños entre 7 y 12 años. El objetivo era conocer la cantidad y calidad del movimiento de los niños mientras jugaban boxeo y tenis en el Nintendo Wii ${ }^{\circledR}$ y esquí y cabeceo en fútbol en el Wii Fit ${ }^{\circledR}$. En una plataforma de fuerza se midió el desplazamiento del centro de presión como un indicador de la cantidad de movimiento, y el movimiento de la pelvis como indicador de la calidad del movimiento. Levac et al. (2010), han encontrado diferencias únicamente en lacantidad ynoen la calidad del movimiento, especialmente en los niños que contaban con experiencias previas utilizando esos aparatos. Por lo tanto, se puede concluir que existe una curva de aprendizaje que permite una mayor cantidad de movimiento a medida que los niños juegan. Esta cantidad de movimiento también puede ser medida para conocer el gasto energético, considerada una variable importante para el control del peso.

Existe evidencia que indica que tanto los padres como los hijos consideran que los videojuegos activos promueven la actividad física y pueden contribuir a reducir la obesidad infantil (Dixon, Maddison, Ni Mhurchu, Jull, Meagher-Lundberg, \& Widdowson, 2010). En un estudio de grupo focal en el que participaron siete niños y cuatro adultos (Dixon et al., 2010), los participantes concluyeron que los videojuegos activos permiten mejorar la cantidad de actividad física y mejorar la condición física ofitness; sin embargo, para los padres, los precios de los videojuegos y la falta de espacio en el hogar son barreras que impiden su utilización.

\section{Efectos sociales}

Se han descrito algunos de los efectos sociales negativos del uso de videojuegos, como por ejemplo, una relación afectiva débil con los padres y personas de su misma edad, o incluso el reforzamiento de estereotipos sociales de tipo racial o sexual (Dickerman, Christensen, \& Kerl-McClain, 2008). En niños de edad preescolar (i.e., jardín de infantes o kindergarten), se ha observado que cuando utilizan videojuegos no les permite desarrollar importantes destrezas sociales debido a que la naturaleza de los videojuegos no les estimula a tener interacciones significativas con otros niños y porque los juegos los distraen, por lo que el efecto en la socialización es perjudicial (Bacigalupa, 2005).
En adolescentes, se ha encontrado que aquellos entre 14 y 19 años de edad que pasan más tiempo frente a una pantalla (e.g., ordenador, T.V., videojuegos), son menos sociables y unidos a sus padres y personas de edades similares (Cummings, \& Vandewater, 2007; Richards, McGee, Williams, Welch, \& Hancox, 2010). Se ha reportado que en promedio, los adolescentes pasan 3.2 horas diarias frente a una pantalla, y que por cada hora extra que estuvieran frente a una pantalla existe un $13 \%$ de probabilidades en el riesgo de que tengan menos apego o unión a sus padres y un $24 \%$ menos de apego para personas de su misma edad (i.e., pares) (Richards et al., 2010).

En la revisión sistemática de literatura de Biddiss e Irwin (2010), se ha indicado que los videojuegos activos podrían ser una herramienta positiva para motivar a los niños a que compitan contra otras personas y amigos, sin importar si lo hacen de manera personal (frente a frente) $o$ a distancia (virtual). Apesar de que esta hipótesis puede parecer atractiva, todavía queda por investigar si este tipo de videojuegos promueven la competencia y el disfrute de los niños, o si de alguna forma permite crear lazos afectivos duraderos que no se basen solamente en la competencia.

Por otra parte, se ha promovido el desarrollo y uso de videojuegos para originar conductas deseables (e.g., aumentar el consumo de vegetables y frutas, utilizar preservativo, aumentar actividad física), así como para rehabilitar alguna discapacidad (Thompson, Baranowski, \& Buday, 2010; Baranowski, Baranowski, Thompson, Buday, Jago, Griffith, Islam, Nguyen, \& Watson, 2011). Existen estudios con resultados positivos en personas que poseen enfermedades crónicas y degenerativas como diabetes mellitus, parálisis cerebral, accidente vascular cerebral, quemaduras, lesión en la espina dorsal, espina bífida, y fibrosis quística (DeShazo, Harris, \& Pratt, 2010; Golomb, McDonald, Warden, Yonkman, Saykin, Shirley, Huber, Rabin, Abdelbaky, Nwosu, BarkatMasih, \& Burdea, 2010; Jannink, van der Wilden, Navis, Visser, Gussinklo, \& Ijzerman, 2008; Deutsch, Borbely, Filler, Huhn, \& Guarrera-Bowlby, 2008; Yavuzer, Senel, Atay, \& Stam, 2008; Haik, Tessone, Nota, Mendes, Raz, Goldan, Regev, Winkler, Mor, Orenstein, \& Hollombe, 2006; O'Connor, Cooper, Fitzgerald, Dvorznak, Boninger, VanSickle, \& Glass, 2000; Widman, McDonald, \& Abresch, 2006; Bingham, Bates, Thompson-Figueroa, \& Lahiri, 2010). En general, este tipo de videojuegos ha permitido mejorar cualidades físicas y psicológicas en los usuarios, y tienen el valor agregado de que se pueden utilizar en el hogar, por lo que no existe la necesidad de desplazarse hasta un hospital o centro de salud. Un aspecto negativo es que estos videojuegos podrían promover el aislamiento social de los usuarios; sin embargo, este tipo de videojuegos se seguirá desarrollando en los próximos años y promete ser importante para el tratamiento de otras patologías (Thompson, Baranowski, \& Buday, 2010).

También se ha sugerido que el uso de medios electrónicos podría promover la interacción social y reducir conductas de riesgo sexual a través de las populares «redes sociales» (e.g., Facebook, MySpace, Twitter, Hi5). Sin embargo, también hay fuertes argumentos acerca de los efectos negativos de estas redes, pues en muchos casos los niños y adolescentes pueden ser vistos y acosados por depredadores sexuales (Strasburger, Jordan, \& Donnerstein, 2010).

\section{Efectos psicológicos}

\subsection{Comportamiento agresivo.}

Bowman y Rotter (1983), han indicado que más del 85\% de los videojuegos contienen temáticas violentas (e.g., muertes, destrucción, violencia). Strasburger, Jordan y Donnerstein (2010), concuerdan e indican que en la actualidad más del 50\% de los videojuegos tienen un contenido violento, y que de éstos más del $90 \%$ han sido clasificados como E10+. De esta forma, se observa que cada vez existe mayor tolerancia a la utilización de videojuegos que promueven la violencia, especialmente en edades tempranas.

Algunos investigadores han sostenido que no existe una asociación causal entre lautilización de videojuegos violentos con el comportamiento agresivo de los niños y adolescentes, y que las publicaciones meta analíticas son sesgadas (Ferguson, 2007; Olson, 2004;Lager \& Brenberg, 
2005; Salonius-Pasternak \& Gelfond, 2005). Sin embargo, en varios meta análisis (Anderson, Shibuya, Ihori, Swing, Bushman, Sakamoto, Rothstein, \& Saleem, 2010; Anderson, 2004; Anderson \& Bushman, 2001; Sherry, 2001), se estudiaron los efectos de los videojuegos violentos sobre conductas agresivas, aspectos cognitivos agresivos, y afecto agresivo, entre otros, y se encontró que la exposición a videojuegos violentos constituye un factor de riesgo causal para conductas agresivas y un comportamiento social reprimido, a pesar de que no se encuentran diferencias entre hombres y mujeres.

Diversos investigadores han observado cambios en el comportamiento luego de la exposición a videojuegos violentos. Por ejemplo, Fleming y Wood (2001), han estudiado en Australia 71 niños con edades entre 8 y 12 años, a quienes sometieron a tres condiciones experimentales. En una ocasión, los niños debían jugar un juego de papel y lápiz. En una segunda ocasión, debían jugar un videojuego no violento; y en una tercera ocasión debían jugar un videojuego violento. Los investigadores midieron el grado de excitación en niños y niñas por medio de la frecuencia cardiaca. Se encontró que la frecuencia cardiaca era mayor luego de jugar el videojuego violento en comparación con las otras dos condiciones experimentales, y que este hallazgo era especialmente dramático para las niñas.

En Irán, se ha realizado un estudio de tipo transversal en una muestra de 444 adolescentes, a quienes se les administró un cuestionario acerca de aspectos sociodemográficos, estado mental, comportamiento agresivo, efectos secundarios percibidos con respecto a los videojuegos de ordenador, y conductas de juego (Allahverdipour, Bazargan, Farhadinasab, \& Moeini, 2010). Se ha encontrado que en promedio, los adolescentes jugaban videojuegos 6.3 horas por semana y que casi la mitad (47\%) jugaban uno o más videojuegos intensamente violentos. También se ha encontrado que las personas que utilizaban videojuegos a edades más tempranas tenían una mayor predisposición a poseer una peor salud mental y que el comportamiento agresivo percibido por los adolescentes era mayor conforme aumentaba el tiempo de exposición al videojuego. También se han encontrado dos hallazgos importantes. Por una parte, los niños mostraron más comportamientos agresivos que las niñas aunque ambos jugaran de forma excesiva. Segundo, los niños que no jugaban videojuegos mostraban los peores resultados en salud mental. Los investigadores han concluido que los jugadores con ordenador propio, que juegan excesivamente, tienen una peor salud mental y una mayor cantidad de comportamientos problemáticos; pero que los niños que no juegan del todo con videojuegos, podrían beneficiarse de una exposición moderada a éstos (Allahverdipour et al., 2010).

Se ha estudiado la asociación entre el número de horas que los niños de escuela primaria pasan sentados viendo televisión y jugando videojuegos con el abuso de sustancias prohibidas (Armstrong, Bush, \& Jones, 2010). Los investigadores han encuestado a 4691 estudiantes de 5to y 6 to grado de escuela primaria en el estado de Kentucky (EEUU), quienes respondieron acerca de sus hábitos con respecto al uso de drogas, alcohol, tiempo que miraban televisión y jugaban videojuegos, y vida en familia. Se ha encontrado que casiun $30 \%$ de los niños reportaron el uso de alguna droga, así como una asociación directa entre el tiempo en que los niños gastaban viendo T.V. y jugando videojuegos (e» $3 \mathrm{~h}$ vs. d» $2 \mathrm{~h}$ ) con el consumo de alcohol y otras drogas. Se ha estimado que los niños que miran la T.V. y que juegan videojuegos e» $3 \mathrm{~h}$ tienen un $48 \%$ más de probabilidades de consumir alcohol y casiel doble de probabilidad de oler algún tipo de droga que aquellos que lohacen $\mathrm{d} \gg 2 \mathrm{~h}$. Sin embargo, una debilidad de este estudio es que no se pudo separar el efecto de ver únicamente T.V. y el efecto de jugar videojuegos.

\subsection{Problemas de atención.}

En un estudio en el que participaron 1323 niños y 210 adolescentes se ha encontrado que la exposición a la T.V. y a los videojuegos estaba asociada a la aparición de problemas atencionales, y que esta asociación también se extiende a la adultez temprana (Swing, Gentile, Anderson, \& Walsh, 2010). El mecanismo fisiológico que podría explicar este hallazgo es el aumento a la exposición de las hormonas del estrés norepinefrina y cortisol (Skosnik, Chatterton, Swisher, \& Park, 2000).
En Turquía, se ha realizado un estudio por medio del cual se pretendía conocer los efectos cognitivos a corto plazo de jugar durante una hora un videojuego en el ordenador. Participaron 101 niños con edades entre 9 y 12 años con y sin problemas psiquiátricos (Tahiroglu, Celik, Avci, Seydaoglu, Uzel, \& Altunbas, 2010). Para evaluar la capacidad cognitiva se ha aplicado la prueba de «Stroop» antes y después del juego. Valores altos del número total de errores y correcciones (NTEC) y la duración total (DT) en la prueba Stroop se consideran como evidencia de falta de atención y mayor deterioro de funciones cognitivas. Los investigadores han encontrado que una hora de videojuego tiene un efecto positivo en la atención a corto plazo en la población general; pero que una vez que se analizan los resultados entre los niños con y sin problemas psiquiátricos, ese efecto desaparece. Así mismo, ese efecto positivo no es evidente en niños que juegan habitualmente esos juegos, pero sí lo es para quienes en el estudio lo jugaron por primera vez. Por lo tanto, se puede concluir que la atención mejora cuando se aprende a jugar un videojuego, que hay una curva de aprendizaje, ya que aumenta la activación cerebral pre frontal y frontal; sin embargo, crónicamente desaparece ese beneficio, por lo que se sugiere que la exposición a largo plazo de videojuegos puede deteriorar la atención en niños, especialmente en personas muy jóvenes con Trastorno por Déficit de Atención e Hiperactividad (TDAH).

\section{Efectos fisiológicos}

\subsection{Respuesta hormonal.}

La respuesta hormonal a los juegos y a los videojuegos ha sido estudiada (Beaven, Ingram, Gill, \& Hopkins, 2010; Gonzalez-Bono, Salvador, Serrano, \& Ricarte, 1999), indicando que en general, se encuentra una tendencia a un aumento en los niveles de testosterona en los jugadores que ganan un juego o videojuego en comparación a los que pierden. Este hallazgo no es tan consistente para el cortisol, pues se han encontrado estudios donde aumentan los niveles post ejercicio (Hébert, Béland, Dionne-Fournelle, Crête, \& Lupien, 2005) y otros cuando hay reducciones o no hay cambios significativos (Denot-Ledunois, Vardon, Perruchet, \& Gallego, 1998; Ivarsson, Anderson, Akerstedt, \& Lindblad, 2009; Paris, Franco, Sodano, Frye, \& Wulfert, 2010; Sharma, Khera, Mohan, Gupta, \& Ray, 2006). Se sabe que la testosterona y el cortisol se liberan durante el ejercicio y que juegan un papel importante en la adaptación del organismo; sin embargo, una elevada exposición crónica a ambas hormonas, especialmente al cortisol, posee efectos negativos en el organismo (e.g., depresión, ansiedad, aumento de glucosa sanguínea, reducción de actividad inmunológica, pérdida de memoria) (Lupien, Nair, Briere, Maheu, Tu, Lemay, McEwen, \& Meaney, 1999).

Para Lager y Brenberg (2005) los videojuegos permiten mejorar habilidades espaciales y tiempo de reacción. Los videojuegos mezclan una combinación poderosa de llamativas escenas visuales y música o sonidos adictivos que en conjunto producen respuestas fisiológicas (e.g., aumento del cortisol). Por ejemplo, en adultos se ha encontrado que quienes utilizan videojuegos con música liberan más cortisol que quienes los usan sin música (Hébert, Béland, Dionne-Fournelle, Crête, \& Lupien, 2005). También se ha reportado que la respuesta de la frecuencia cardíaca es mayor en hombres y que la presión arterial es mayor en las mujeres cuando juegan videojuegos (Tafalla, 2007).

\subsection{Gasto energético.}

La premisa de investigación es conocer si los videojuegos (activos vs. no activos) permiten un gasto energético similar al del juego libre; es decir, sin aparatos electrónicos. Esto es principalmente importante para niños y adolescentes con sobrepeso y obesidad. En general, se ha encontrado que en comparación con videojuegos no activos tradicionales, en los que se involucra únicamente la destreza óculo-manual y la persona se encuentra sentada la mayor parte del tiempo, los videojuegos activos aumentan considerablemente el gasto energético (medido en Kcals, kJ, MET, o $\mathrm{mL} \mathrm{O}_{2}$ ) y otras variables fisiológicas como la presión arterial sistólica y diastólica, y la frecuencia respiratoria (Ambrosetti, \& Saltarelli, 1995; Borusiak, Bouikidis, Liersch, \& Russell, 2008; Denot-Ledunois, 
Vardon, Perruchet, \& Gallego, 1998; Foley \& Maddison, 2010; Segal \& Dietz, 1991), posiblemente debido a una liberación de hormonas del estrés como la epinefrina, la testosterona y el cortisol, que en conjunto estimulan al excitación y la emoción.

El aspecto del posible aumento en el gasto energético es importante porque existe información contradictoria acerca del consumode alimentos (i.e., ingesta de energía) cuando se juegan videojuegos pasivos y activos. Recientemente, se haencontrado que no existe una diferencia significativa en el consumo de alimentos (meriendas, no comidas principales) cuando niños de 9 a 13 años utilizan videojuegos pasivos (i.e., sentados) o cuando utilizan videojuegos activos (Mellecker, Lanningham-Foster, Levine, \& McManus, 2010).

A pesar de que se ha promovido el uso de diferentes videojuegos para estimular la pérdida de peso y el aumento en el gasto energético; recientemente se ha encontrado que el interés en los videojuegos decae después de 12 semanas cuando personas obesas los utilizan en un ambiente clínico (Radon, Furbeck, Thomas, Siegfried, Nowak, \& von Kries, 2011).

Penko y Barkley (2010), han estudiado un grupo de 11 niños delgados y otro grupo de 13 niños obesos entre 8 y 12 años de edad. Los participantes debían jugar un videojuego de boxeo en Nintendo $\mathrm{Wii}^{\circledR}$, caminar en una banda sin fin, y utilizar un juego pasivo. Se ha medido el gasto energético en cada modalidad de actividad y la motivación para jugar. Los investigadores han encontrado mayores gastos energéticos cuando los niños jugaban con Nintendo $\mathrm{Wii}^{\circledR}$ que cuando lo hacían con un juego pasivo. Además, han reportado que los niños delgados estaban más motivados al jugar con el Nintendo que cuando lo hacían con el juego pasivo, mientras que los niños obesos estaban igualmente motivados al jugar con ambos tipos de juegos. Sit, Lam y McKenzie (2010), también han realizado una investigación en Hong Kong en la que participaron 140 niños obesos y no obesos con edades entre los 9 y los 12 años. El propósito del estudio era determinar preferencias en el tipo de videojuego y se encontró que los niños prefieren los juegos más interactivos en comparación con los pasivos, y que el gasto energético alcanzado pudo categorizarse como de moderada a alta intensidad.

De manera similar, White, Schofield y Kilding (2011), han realizado un estudio con 26 niños de 11 años de edad en Nueva Zelandia para conocer el gasto energético durante la realización de videojuegos activos en Nintendo Wii ${ }^{\circledR}$ (i.e., bolos, boxeo, tenis, esquí y ejercicios aeróbicos de «step»), actividades sedentarias (i.e., descansar sentados, ver T.V., o jugar un juego pasivo), caminar y correr. Durante todas las actividades, los investigadores han medido el gasto energético y han encontrado que los videojuegos activos aumentaban el gasto energético significativamente en comparación con el reposo y con los juegos pasivos, pero no comparados con caminar o correr (Gráfico 1). Los investigadores han concluido que los videojuegos activos no son lo suficientemente intensos como para contribuir con la recomendación actual de $60 \mathrm{~min}$ de actividad física diaria moderada a vigorosa.

En una revisión sistemática de literatura reciente (Biddiss \& Irwin, 2010), se ha investigado el gasto energético y los cambios en los patrones de actividad física asociados con los videojuegos activos en que participan

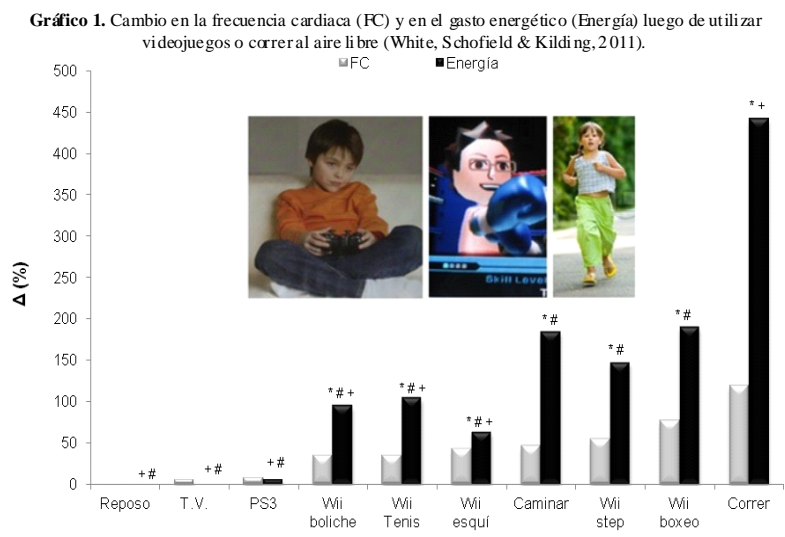

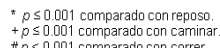

los niños (edad d» 21 años). Se ha encontrado que los niveles de actividad física durante los videojuegos activos alcanzaban en promedio un aumento del $222 \%$ en gasto energético en comparación a cuando los niños estaban en reposo; sin embargo, existe una alta variabilidad (desviación típica de $\pm 100 \%$ ). También han reportado aumentos del $64 \%$ en la frecuencia cardiaca desde el reposo, lo que indica que en general, los videojuegos activos pueden considerarse como actividades de intensidad liviana a moderada, pero no se les puede considerar como vigorosas a pesar de estudios recientes (Sit et al., 2010). Es decir, en general, los niños no alcanzan ni superan el $80 \%$ de su frecuencia cardiaca máxima $\left(\mathrm{FC}_{\mathrm{max}}\right)$, la cual se podría considerar como beneficiosa para mejorar la capacidad aeróbica (Baquet, Van Praagh, \& Berthoin, 2003), o el 85-90\% de su $\mathrm{FC}_{\text {maxx }}$ considerado adecuado para mejorar la calidad del sueño (Dworak, Wiater, Alfer, Stephan, Hollmann, \& Strüder, 2008). Este hallazgo es especialmente verdadero para los videojuegos que involucran el tren inferior en comparación con únicamente el tren superior, pues se encontró que existen diferencias estadísticamente significativas en el gasto energético cuando únicamente se utiliza el tren superior en comparación con el tren inferior.

\subsection{Vista y atención visual}

Con respecto a la salud visual, se ha comparado niños de 6 a 14 años de edad (731 personas con miopía y 587 personas con visión normal) que utilizaban videojuegos y realizaban otras actividades (Jones-Jordan, Mitchell, Cotter, Kleinstein, Manny, Mutti, Twelker, Sims, \& Zadnik, 2011). Los investigadores han encontrado que el número de horas semanales que las personas leían y utilizaban videojuegos o el ordenador eran significativamente mayores en las personas con miopía que en las personas con visión normal. De la misma manera, se ha reportado que las personas miopes practicaban una menor cantidad de horas semanales de actividades deportivas al aire libre que las personas con visión normal.

Por otra parte, Dye y Bavelier (2010), han evaluado tres aspectos de la atención visual en niños acostumbrados a jugar videojuegos y en niños no acostumbrados a jugarlos. Las edades de los 114 niños y niñas estaban entre los 7 y los 17 años. Primero, se ha medido la habilidad para distribuir la atención en un campo visual para la búsqueda de un objetivo. Luego, se ha medido el tiempo que era necesario para recuperar la atención visual después de encontrar el objetivo. Finalmente, se ha medido el número de objetos que pueden ser simultáneamente buscados. Los investigadores han encontrado que los niños y adolescentes que jugaban a menudocon videojuegos de acción mostraron mejores puntajes en todas las pruebas que aquellos que no jugaban regularmente. Este hallazgo sugiere que los niños que juegan con videojuegos de acción alcanzan patrones de atención visual que solo se alcanzan en etapas más maduras del desarrollo en comparación con los niños que no juegan regularmente.

\subsection{Sistema nervioso central.}

Un posible efecto negativo de la exposición prolongada a los videojuegos es la llamada «epilepsia fotosensible», la cual ha sido estudiada desde el año 1950 en personas que sufrieron episodios mientras miraban televisión (Harding \& Harding, 2010). A pesar de que la incidencia de la epilepsia fotosensible es baja en la población general (i.e., 1 persona por cada 4000 habitantes), se ha encontrado que la incidencia es cinco veces mayor en adolescentes entre 7 y 19 años de edad. Se sabe que las mujeres son más fotosensibles que los hombres (Harding \& Harding, 2010); sin embargo, se ha reportado que un $75 \%$ de los hombres que utilizan videojuegos sufren de episodios epilépticos, en comparación con un 25\% de las mujeres (Graf, Chatrian, Glass, \& Knauss, 1994), y que uno de los mecanismos que explican los episodios es la fotosensibilidad (Ferrie, De Marco, Gruanewald, Giannakodimos, \& Panayiotopoulos, 1994; Fylan, Harding, Edson, \& Webb, 1999).

Finalmente, en una revisión narrativa de 36 investigaciones (Cain \& Gradisar, 2010), se evaluó el impacto de la exposición a medios electrónicos (i.e., T.V., videojuegos, ordenador) en la calidad del sueño de los niños y adolescentes (d» 17 años). Los investigadores han encontrado que en general, los niños y adolescentes que pasan más tiempo jugando 
videojuegos empeoran sus patrones de sueño, especialmente el tiempo total dormido y el tiempo total en la cama. Este hallazgo contrasta con estudios en donde se ha observado que el ejercicio físico intenso promueve una mejor calidad de sueño en niños de 12 años de edad (Dworak, Wiater, Alfer, Stephan, Hollmann, \& Strüder, 2008).

\section{Conclusiones}

Al finalizar esta revisión acerca de los efectos del uso de videojuegos en variables sociales, psicológicas y fisiológicas se llega a las siguientes conclusiones:

1. La industria de los videojuegos es multimillonaria y se espera que siga creciendo en los próximos años.

2. Los niños, adolescentes y adultos gastan una importante cantidad de tiempo frente a la pantalla de un dispositivo electrónico, y no se espera que esta situación cambie en los próximos años debido a la globalización y el acceso a estos dispositivos.

3. El juego tradicional y el proporcionado por los videojuegos seguirá siendo importante en todas las etapas del desarrollo de niños y adolescentes; por lo que se debe controlar la exposición y el contenido de éstos.

4. La evidencia científica acerca de la temática de los videojuegos es algunas veces contradictoria. Se deben realizar mayor cantidad de estudios con sólidos diseños de investigación.

5. Jugar un videojuego activo es más beneficioso que ser sedentario. Sin embargo, los videojuegos activos son más beneficiosos que los videojuegos pasivos.

6. El juego al aire libre es más beneficioso que el proporcionado por los videojuegos.

\section{Referencias}

Aleks Krotoski, A. (2010). Serious fun with computer games. Nature, 466(7307), 695.

Allahverdipour, H., Bazargan, M., Farhadinasab, A., \& Moeini, B. (2010) Correlates of video games playing among adolescents in an Islamic country. BMC Public Health, 10, 286-292.

Ambrosetti, L., \& Saltarelli, W.A. (1995). The physiological response of obese vs. non-obese children to playing action pad video games. Pediatric Exercise Science, 7, 104.

Anderson, C. A. (2004). An update on the effects of playing violent video games. Journal of Adolescence, 27, 113-122.

Anderson, C. A., \& Bushman, B. J. (2001). Effects of violent video games on aggressive behavior, aggressive cognition, aggressive effect, physiological arousal, and prosocial behavior: A meta-analytic review of the scientific literature. Psychological Science, 12(5), 353-359.

Anderson, C. A., Shibuya, A., Ihori, N., Swing, E. L., Bushman, B. J., Sakamoto, A., Rothstein, H. R., \& Saleem, M. (2010). Violent video game effects on aggression, empathy, and prosocial behavior in eastern and western countries: A meta-analytic review. Psychological Bulletin, 136(2), 151-173.

Armstrong, K. E., Bush, H. M., \& Jones, J. (2010). Television and video game viewing and its association with substance use by Kentucky elementary school students, 2006. Public Health Reports, 125(3), 433-440.

Bacigalupa, C. (2005). The use of video games by kindergartners in a family child care setting. Early Childhood Education Journal, 33(1), 25-30.

Baquet, G., Van Praagh, E., \& Berthoin, S. (2003). Endurance training and aerobic fitness in young people. Sports Medicine, 33(15), 1127 1143.

Baranowski, T., Baranowski, J., Thompson, D., Buday, R., Jago, R., Griffith, M. J., Islam, N., Nguyen, N., \& Watson, K. B. (2011). Video game play, child diet, and physical activity behavior change a randomized clinical trial. American Journal of Preventive Medicine, 40(1), 3338

Bavelier, D., Green, C. S., \& Dye, M. W. (2010). Children, wired: for better and for worse. Neuron, 67(5), 692-701.

Beaven, C. M., Ingram, J. R., Gill, N. D., \& Hopkins, W. G. (2010). Ultradian rhythmicity and induced changes in salivary testosterone. European Journal of Applied Physiology, 110(2), 405-143.

Belli, S., \& López Raventós, C. (2008). Breve historia de los videojuegos. Athenea Digital, 14, 159-179.
Biddiss, E., \& Irwin, J. (2010). Active video games to promote physical activity in children and youth: A systematic review. Archives of Pediatric and Adolescent Medicine, 164(7), 664-672.

Bingham, P. M., Bates, J. H., Thompson-Figueroa, J., \& Lahiri, T. (2010) A breath biofeedback computer game for children with cystic fibrosis. Clinical Pediatrics, 49(4), 337-342.

Borusiak, P., Bouikidis, A., Liersch, R., \& Russell, J. B. (2008). Cardiovascular effects in adolescents while they are playing video games: A potential health risk factor?. Psychophysiology, 45(2), 327-332.

Bowman, R. P., \& Rotter, J. C. (1983). Computer games: Friend or foe? Elementary School Guidance and Counseling, 18, 25-34.

Cain, N., \& Gradisar, M. (2010). Electronic media use and sleep in schoolaged children and adolescents: A review. Sleep Medicine, 11(8), 735742.

Carson, V., Pickett, W., \& Janssen, I. (2010). Screen time and risk behaviors in 10- to 16-year-old Canadian youth. Preventive Medicine, 52, 99103.

Chacón Araya, Y. (2005). Una revisión crítica del concepto de creatividad. Revista Electrónica Actualidades Investigativas en Educación, 5(1), $1-30$.

Choo, H., Gentile, D. A., Sim, T., Li, D., Khoo, A., \& Liau, A. K. (2010). Pathological video-gaming among Singaporean youth. Annals of the Academy of Medicine, Singapore, 39(11), 822-828.

Cummings, H. M., \& Vandewater, E. A. (2007). Relation of adolescent video game play to time spent in other activities. Archives of Pediatrics \& Adolescent Medicine, 161(7), 684-689.

Denot-Ledunois, S., Vardon, G., Perruchet, P., \& Gallego, J. (1998). The effect of attentional load on the breathing pattern in children. International Journal of Psychophysiology, 29(1), 13-21.

DeShazo, J., Harris, L., \& Pratt, W. (2010). Effective intervention or child's play? A review of video games for diabetes education. Diabetes technology \& Therapeutics, 12(10), 815-822.

Deutsch, J.E., Borbely, M., Filler, J., Huhn, K., \& Guarrera-Bowlby, P. (2008). Use of a low-cost, commercially available gaming console (Wii) for rehabilitation of an adolescent with cerebral palsy. Physical Therapy, 88(10), 1196-1207.

Dickerman, C., Christensen, J., \& Kerl-McClain, S.B. (2008). Big breasts and bad guys: Depictions of gender and race in video games. Journal of Creativity in Mental Health, 3(1), 20-29.

Dixon, R., Maddison, R., Ni Mhurchu, C., Jull, A., Meagher-Lundberg, P., \& Widdowson, D. (2010). Parents' and children's perceptions of active video games: a focus group study. Journal of Child Health Care, 14(2), 189-199.

Dworak, M., Wiater, A., Alfer, D., Stephan, E., Hollmann, W., \& Strüder, H. K. (2008). Increased slow wave sleep and reduced stage 2 sleep in children depending on exercise intensity. Sleep Medicine, 9(3), 266272.

Dye, M. W., \& Bavelier, D. (2010). Differential development of visual attention skills in school-age children. Vision Research, 50(4), 452459.

Eagle, T. F., Gurm, R., Goldberg, C. S., DuRussel-Weston, J., Kline-Rogers, E., Palma-Davis, L., Aaronson, S., Fitzgerald, C. M., Mitchell, L. R., Rogers, B., Bruenger, P., Jackson, E. A., \& Eagle, K. A. (2010). Health status and behavior among middle-school children in a midwest community: what are the underpinnings of childhood obesity? American Heart Journal, 160(6), 1185-1189.

Entertainment Software Rating Board. (2010). Frequently asked questions. Con acceso en línea (31/12/2010): http://www.esrb.org/ratings/ faq.jsp\#1

Estes, S. G., \& Mechikoff, R. A. (1999). Knowing human movement. Boston, MA: Allyn and Bacon.

Ferguson, C. J. (2007). Evidence for publication bias in video game violence effects literature: A meta-analytic review. Aggression and Violent Behavior, 12, 470-482.

Ferrie, D.D., De Marco, P., Gruanewald, R.A., Giannakodimos, S., \& Panayiotopoulos, C.P. (1994). Video game induced seizures. Journal of Neurology, Neurosurgety, and Psychiatry, 57, 925-931.

Fleming, M. J., \& Wood, D. J. R. (2001). Effects of violent versus nonviolent video games on children's arousal, aggressive mood, and positive mood. Journal of Applied Social Psychology, 31(10), 20472071

Foley, L., \& Maddison, R. (2010). Use of active video games to increase physical activity in children: a (virtual) reality? Pediatric Exercise Science, 22(1), 7-20. 
Fylan, F., Harding, G.F.A., Edson, A.S., \& Webb, R.M. (1999). Mechanisms of video-game epilepsy. Epilepsia, 40(Suppl. 4), 28-30.

Golomb, M. R., McDonald, B. C., Warden, S. J., Yonkman, J., Saykin, A J., Shirley, B., Huber, M., Rabin, B., Abdelbaky, M., Nwosu, M. E., Barkat-Masih, M., \& Burdea, G. C. (2010). In-home virtual reality videogame telerehabilitation in adolescents with hemiplegic cerebral palsy. Archives of Physical Medicine and Rehabilitation, 91(1), 1-8.

Gonzalez-Bono, E., Salvador, A., Serrano, M.A., \& Ricarte, J. (1999). Testosterone, cortisol, and mood in a sports team competition. Hormones and Behavior 35, 55-62.

Graf, W.D., Chatrian, G.E., Glass, S.T., \& Knauss, T.A. (1994). Video game-related seizures: A report on 10 patients and a review of the literature. Pediatrics, 93, 551-556.

Haik, J., Tessone, A., Nota, A., Mendes, D., Raz, L., Goldan, O., Regev, E., Winkler, E., Mor, E., Orenstein, A., \& Hollombe, I. (2006). The use of video capture virtual reality in burn rehabilitation: the possibilities. Journal of Burn Care \& Research, 27(2), 195-197.

Harding, G. F., \& Harding, P. F. (2010). Photosensitive epilepsy and image safety. Appl Ergon, 41(4), 504-508.

He, M., Piche, L., Beynon, C., \& Harris, S. (2010). Screen-related sedentary behaviors: Children's and parents' attitudes, motivations, and practices. Journal of Nutrition Education and Behavior, 42(1), 17-25.

Hébert, S., Béland, R., Dionne-Fournelle, O., Crête, M., \& Lupien, S. J. (2005). Physiological stress response to video-game playing: The contribution of built-in music. Life Sciences, 76, 2371-2380.

Hofferth, S. L. (2010). Home media and children's achievement and behavior. Child Development, 81(5), 1598-1619.

Holtz, P., \& Appel, M. (2011). Internet use and video gaming predict problem behavior in early adolescence. Journal of Adolescence, 34(1), 49-58

Hoyos Cillero, I., \& Jago, R. (2010). Systematic review of correlates of screen-viewing among young children. Preventive Medicine, 51(1), 3-10.

Ivarsson, M., Anderson, M., Akerstedt, T., \& Lindblad, F. (2009). Playing a violent television game does not affect saliva cortisol. Acta Paediatrica, 98(6), 1052-1053.

Jannink, M.J., van der Wilden, G.J., Navis, D.W., Visser, G., Gussinklo, J., \& Ijzerman, M. (2008). A low-cost video game applied for training of upper extremity function in children with cerebral palsy: A pilot study. CyberPsychology \& Behavior, 11(1), 27-32.

Jones-Jordan, L. A., Mitchell, G. L., Cotter, S. A., Kleinstein, R. N., Manny, R. E., Mutti, D. O., Twelker, J. D., Sims, J. R., \& Zadnik, K. (2011). Visual activity before and after the onset of juvenile myopia. Investigative Ophthalmology \& Visual Science, 52, 1841-1850.

King, D., Delfabbro, P., \& Griffiths, M. (2010). The convergence of gambling and digital media: implications for gambling in young people. Journal of Gambling Studies, 26(2), 175-87.

Lager, A., \& Brenberg, S. (2005). Health Effects of Video and Computer Game Playing: A systemic review of scientific studies. Suecia: National Swedish Public Health Institute.

Levac, D., Pierrynowski, M. R., Canestraro, M., Gurr, L., Leonard, L., \& Neeley, C. (2010). Exploring children's movement characteristics during virtual reality video game play. Human Movement Sciences 29(6), 1023-1038.

Lupien, S.J., Nair, N.P., Briere, S., Maheu, F., Tu, M.T., Lemay, M., McEwen, B.S., \& Meaney, M. J. (1999). Increased cortisol levels and impaired cognition in human aging: Implication for depression and dementia in later life. Reviews in Neuroscience, 10(2), 117-139.

Moncada-Jiménez, J. (2005). 2005: Año Internacional del Deporte y la Educación Física. Revista Educación, 29(2), 233-247.

Mossle, T., Kleimann, M., Rehbein, F., \& Pfeiffer, C. (2010). Media use and school achievement-boys at risk? British Journal ofDevelopmental Psychology, 28(Pt. 3), 699-725.

O’Connor, T.J., Cooper, R.A., Fitzgerald, S.G., Dvorznak, M. J., Boninger, M.L., VanSickle, D.P., \& Glass, L. (2000). Evaluation of a manual wheelchair interface to computer games. Neurorehabilitation \& Neural Repair, 14(1), 21-31.

Olson, C. (2004). Media violence research and youth violence data: Why do they conflict?», Academic Psychiatry, 28(2), 144-150.

Paris, J. J., Franco, C., Sodano, R., Frye, C. A., \& Wulfert, E. (2010). Gambling pathology is associated with dampened cortisol response among men and women. Physiology \& Behavior, 99(2), 230-233.

Pellegrini, A. D. (2010). Games and play mean different things in an educational context. Nature, 467(7311), 27.

Penko, A. L., \& Barkley, J. E. (2010). Motivation and physiologic responses of playing a physically interactive video game relative to a sedentary alternative in children. Annals of Behavioral Medicine, $39(2), 162-169$

Radon, K., Furbeck, B., Thomas, S., Siegfried, W., Nowak, D., \& von Kries, R. (2011). Feasibility of activity-promoting video games among obese adolescents and young adults in a clinical setting. Journal of Science and Medicine in Sport, 14(1), 42-45.

Ray, M., \& Jat, K. R. (2010). Effect of electronic media on children. Indian Pediatrics, 47(7), 561-568.

Rey-Lopez, J. P., Vicente-Rodriguez, G., Ortega, F. B., Ruiz, J. R., MartinezGomez, D., De Henauw, S., Manios, Y., Molnar, D., Polito, A., Verloigne, M., Castillo, M. J., Sjostrom, M., De Bourdeaudhuij, I., \& Moreno, L. A. (2010). Sedentary patterns and media availability in European adolescents: The HELENA study. Preventive Medicine, 51(1), 50-55

Richards, R., McGee, R., Williams, S. M., Welch, D., \& Hancox, R. J. (2010). Adolescent screen time and attachment to parents and peers. Archives of Pediatrics \& Adolescent Medicine, 164(3), 258-262.

Rosser, J.C., Lynch, P.J., Cuddihy, L., Gentile, D.A., Klonsky, J., \& Merrell, R. (2007). The impact of video games on training surgeons in the $21^{\text {st }}$ Century. Archives of Surgery, 142, 181-186.

Salonius-Pasternak, D. E., \& Gelfond, H. S. (2005). The next level of research on electronic play: Potential benefits and contextual influences for children and adolescents. Human Technology: An Interdisciplinary Journal on Humans in ICT Environments, 1(1), 522.

Scarlett, W. G., Naudeau, S., Ponte, I. C., \& Salonius-Pasternak, D. E. (2004). Children's play. Thousand Oaks, CA: Sage Publications.

Segal, K. R., \& Dietz, W. H. (1991). Physiologic responses to playing a video game. American Journal of Diseases in Children, 145(9), 10341036.

Sharif, I., Wills, T. A., \& Sargent, J. D. (2010). Effect of visual media use on school performance: A prospective study. Journal of Adolescent Health, 46(1), 52-61.

Sharma, R., Khera, S., Mohan, A., Gupta, N., \& Ray, R. B. (2006). Assessment of computer game as a psychological stressor. Indian Journal of Physiology and Pharmacology, 50(4), 367-374.

Sherry, J. L. (2001). The effects of violent video games on aggression: A meta-analysis. Human Communication Research, 27(3), 409-431.

Sit, C. H., Lam, J. W., \& McKenzie, T. L. (2010). Direct observation of children's preferences and activity levels during interactive and online electronic games. Journal of Physical Activity \& Health, 7(4), 484 489 . 4 th

Skosnik, P. D., Chatterton, R. T. Jr, Swisher, T., \& Park, S. (2000). Modulation of attentional inhibition by norepinephrine and cortisol after psychological stress. International Journal of Psychophysiology, 36(1), 59-68.

Strasburger, V. C., Jordan, A. B., \& Donnerstein, E. (2010). Health effects of media on children and adolescents. Pediatrics, 125(4), 756-767.

Swing, E. L., Gentile, D. A., Anderson, C. A., \& Walsh, D. A. (2010). Television and video game exposure and the development of attention problems. Pediatrics, 126(2), 214-221.

Tafalla, R. J. (2007). Gender differences in cardiovascular reactivity and game performance related to sensory modality in violent video game play. Journal of Applied Social Psychology, 37(9), 20082023 .

Tahiroglu, A. Y., Celik, G. G., Avci, A., Seydaoglu, G., Uzel, M., \& Altunbas, H. (2010). Short-term effects of playing computer games on attention. Journal of Attention Disorders, 13(6), 668-676.

The Entertainment Software Association. (2011). Industry facts. Con acceso en línea (31/12/2010): http://www.theesa.com/facts/index.asp

Thompson, D., Baranowski, T., \& Buday, R. (2010). Conceptual model for the design of a serious video game promoting self-management among youth with type 1 diabetes. Journal of Diabetes Science and Technology, 4(3), 744-749.

White, K., Schofield, G., \& Kilding, A. E. (2011). Energy expended by boys playing active video games. Journal of Science and Medicine in Sport, 14(2), 130-134.

Widman, L.M., McDonald, C.M., \& Abresch, R.T. (2006). Effectiveness of an upper extremity exercise device integrated with computer gaming for aerobic training in adolescents with spinal cord dysfunction. The Journal of Spinal Cord Medicine, 29(4), 363-370.

Yavuzer, G., Senel, A., Atay, M.B., \& Stam, H.J. (2008). «PlayStation EyeToy Games» improve upper extremity-related motor functioning in subacute stroke: A randomized controlled clinical trial. European Journal of Physical and Rehabilitation Medicine, 44(3), 237-244. 